\title{
EDUCACIÓN RURAL EN AMÉRICA LATINA Y EL CARIBE: UN DESAFÍO HACIA EL FUTURO
}

\author{
Ph.D. Juan Caliva Esquivel*
}

Estamos ante una descripción de los principales aspectos que han venido incidiendo en la marginalidad de las zonas rurales respecto a los beneficios que debe proveer un concepto de desarrollo integral.

Partiendo de una valoración del concepto de desarrollo sostenible, el autor plantea la necesidad de que se considere como "sujeto" del desarrollo a todos los habitantes de las zonas rurales, lo cual implica contemplar el análisis, las dimensiones social, económica, política y ecológica de forma tal que contribuyan a potenciar el desarrollo de los espacios runales.

La falta de voluntad política -desde la perspectiva del autor-prevaleciente en América Latina, por parte de gobiernos, ha impedido cambiar a las mujeres y los hombres del campo, pues pareciera ser que son se quiere que aprendan, se eduquen y se incorporen a las demás fuerzas sociales en igualdad de condiciones.
A description is presented of the main aspects that have been affecting the marginality of rural areas regarding the benefits that a comprehensive development concept should provide. Based on an assessment of the concept of sustainable development, the author has proposed that the "subject" of development must be all the inhabitants of rural areas. This implies taking into account the analysis as well as social, economic, political and ecological dimensions so that they: can contribute to strengthening the development of rural areas.

The lack of political will-from the author's point of view-prevailing in Latin America among governmental authorities, has made it impossible to transform men and women in rural areas. It would appear that they should have an equal opportunity to learn, receive education and become part of the rest of the social forces.

* El autor obtuvo en grado "of Philosophy (Ph.D.) mayor in Education fon Iowa State University", equiparado a doctor en Educación por la Universidad de Costa Rica.

Labora como especialista en educación agrícola en el Instituto Interamericano de Cooperación para la Agricultura (IICA - OEA). jcaliva@iica.ac.cr 


\section{Introducción}

La situación de la educación en las zonas rurales de América Latina parece ser la consecuencia de un conjunto de situaciones históricas que han venido modelando las caracteristicas específicas que hoy posee.

Este conjunto de situaciones determina los procesos de cambio dentro de ese segmento importante del sistema social latinoamericano. Por estas razones, todo esfuerzo de planificación y ejecución de la educación para el medio rural debería tener en cuenta las múltiples conexiones que los distintos elementos de nuestro sistema social tienen entre sí, así como la diversidad de situaciones de ruralidad, de niveles y modalidades de desarrollo y los distintos procesos históricos que condujeron a esta situación.

Existe sin duda en América Latina un hecho político evidente: los diferentes intentos de planificación social y económica. así como las reformas educacionales se han preocupado fundamentalmente por el desarrollo de las zonas urbanas, relegando las rurales a un segundo plano.

Los fenómenos de la industrialización y de la concentración humana en las ciudades han provocado una presión sociopolítica mayor en estas zonas para conseguir un mejoramiento en los niveles de vida y una búsqueda de soluciones a los problemas sociales derivados de dichos fenómenos. En ese contexto se han llevado a cabo, por ejemplo, soluciones para las poblaciones marginadas en las grandes ciudades y zonas metropolitanas y acciones destinadas a la preparación de mano de obra calificada para la industria en todas sus diferentes áreas. Como resultado de estas políticas hemos visto desarrollarse múltiples zonas francas y parques industriales, junto con amplios programas vocacionales de educación técnico-profesional para la calificación y el mejoramiento de la mano de obra laboral de trabajadores en servicio. Esta realidad deberá ser considerada en todos los esfuerzos futuros del planeamiento de la educación para los habitantes de medio rural latinoamericano, aprovechando esta fuente de experiencias logradas en las zonas urbanas con miras a enfrentar acciones en el porvenir.

Dentro de todo este contexto resultan explicables las limitaciones existentes en cuanto a un conocimiento adecuado de las características y de la problemática de cada una de las zonas rurales en las diferentes regiones de América Latina. La escasez de informaciones confiables, acompañada de la falta de técnicas y metodologías apropiadas para la investigación y programación en estas zonas, dificultan y hasta impiden la elaboración de análisis científicos sobre las relaciones que se operan entre las diferentes variables que inciden genuina y legítimamente en el desarrollo de las comunidades rurales; así como la traducción de sus necesidades en programas específicos de acción productiva. Todo lo 
anterior repercute en un desconocimiento real de los diferentes niveles de desarrollo y de calidad de vida predominantes en las zonas rurales latinoamericanas y del papel que la educación y la tecnología deben jugar en este medio social.

El mejoramiento de las condiciones de vida en los espacios rurales, en su acepción más amplia y de mayor alcance, se aproxima al concepto de desarrollo rural sostenible, el cual implica el desarrollo de sus dimensiones sociales, económicas, políticas y ecológicas y reconoce como "sujeto" a todos los habitantes de las áreas rurales. Un alcance restringido privando los conceptos de permanencia, sostenibilidad y auto propulsión, pierde potencia para contribuir a impulsar de manera efectiva el desarrollo de los espacios rurales.

Las razones expuestas obligan a la búsqueda de un desarrollo sostenible para los espacios rurales, con el objetivo del logro permanente de mejores condiciones de vida y la creación de capacidades para la competitividad.

El apoyo al mejoramiento de las condiciones de vida en los espacios rurales deberá centrarse en el desarrollo de las capacidades humanas de los habitantes de espacios rurales por medio de procesos de educación y capacitación que incremente su capacidad para aumentar el desarrollo de las telecomunicaciones y de la capacidad empresarial y de gestión: en el fortalecimiento de las instituciones públicas y privadas relacionadas; en el apoyo a las organizaciones económicas y cívicas de las sociedades rurales, en una adecuada comercialización de bienes y servicios y en la diversificación y en el empleo.

\section{Antecedentes: marginalidad y dependencia de las zonas rurales}

Inicialmente puede afirmarse que las zonas rurales de América Latina se caracterizan, en general, por su marginalidad y dependencia en relación con las urbanas, las que han concentrado las mayores inversiones asegurando, de esta manera, más altos niveles de crecimiento socioeconómico. Por otra parte, esta política ha llevado a crear grandes e injustos desequilibrios entre zonas urbanas y rurales en cuanto a la distribución de bienes y servicios, generándose la vergonzante situación de ciudadanos de primera, segunda y tercera categoría, según tengan o no, acceso a éstos.

Hacia el final del siglo, diferentes diagnósticos nos indican que la situación rural en muchos espacios rurales del continente con un panorama que a continuación se describe: campesinos y trabajadores rurales sin tierra están subalimentados, pobre y deficientemente alojados y sin cuidados médicos y educación. Mujeres y jóvenes sufren con mayor impacto la influencia de las condiciones adversas del medio rural. solución de los problemas. Recordemos que el costo social de la contribución del sector agrícola al desarrollo de los 
Inicialmente puede afirmarse que las zonas rurales de América Latina se caracterizan, en general, por su marginalidad y dependencia en relación con las urbanas, las que han concentrado las mayores inversiones asegurando, de esta manera, más altos niveles de crecimiento socioeconómico. Por otra parte, esta política ha llevado a crear grandes e injustos desequilibrios entre zonas urbanas y rurales en cuanto a la distribución de bienes y servicios, generándose la vergonzante situación de ciudadanos de primera, segunda y tercera categoria, según tengan o no, acceso a éstos. países, como consecuencia de estos problemas, la población rural se ve cada vez más estimulada a emigrar de su contexto natural para incorporarse a zonas urbanas en las que espera encontrar mejores niveles de vida. Es un derecho humano legítimo al que deben apelar los habitantes rurales cuando la miseria y las infimas condiciones humanas así lo demandan. Naturalmente, este fen6meno humano migratorio no se da con la misma intensidad en todas las diferentes zonas rurales de América Latina.

Es la falta de voluntad política que ha prevalecido por parte de los gobiernos y políticos latinoamericanos, ya que no hay interés en cambiar a los hombres de campo. No se quiere que aprendan, que se eduquen, que se incorporen a las demás fuerzas del país con pleno juicio de sus responsabilidades humanas y cívicas — derechos y deberes- para decidir lo mejor para sus localidades, regiones y para la nación, en general. Hay por el contrario interés en mantenerlos analfabetos, privados del pan de la enseñanza, sin aprender oficios apropiados, sin haber desarrollado destrezas y habilidades, y sobre todo, hay interés en que no aprendan a pensar, a razonar y a convertirse en ciudadanos responsables, en el más amplio sentido de la palabra. Por supuesto, ellos constituyen una masa poblacional numéricamente importante para ser utilizada y manipulada como marioneta en los procesos electorales y en todas aquellas circunstancias que los casos requieran en favor del gobierno, de los políticos y de los intereses y sectores poderosos de cada país.

La mayoría de los indicadores disponibles señalan que la población rural latinoamericana vive en condiciones de una pobreza relativa, lindando con niveles de miseria absoluta, cuyas consecuencias tangibles constituyen un reto para solucionar a corto, mediano y largo plazo. 


\section{Centralismo y ausencia de participación: causas del fracaso y bajo rendimiento de la educación rural}

La falta de participación de la población rural, tanto en las decisiones de carácter económico como en las de carácter social y político influye, naturalmente, en la ausencia de una organización y un sentido comunitario, junto a la dispersión de las poblaciones rurales y a la escasez de medios de comunicación. Como consecuencia, las políticas de desarrollo definidas para el medio rural favorecen la centralización predominante en casi todos los sectores gubernamentales, especialmente en el sector de la educación. Esta centralización dificulta la integración de este sector al desarrollo nacional y su adecuación a las necesidades concretas.

Las actividades educativas se desarrollan en muchos casos, desarticulada, ineficiente y discriminadamente, actuando de forma dispersa cada una de las instituciones que llevan a cabo labores educativas escolares o extraescolares en el medio rural.

Los contenidos son concebidos en la mayoria de los casos a partir de situaciones ajenas al ámbito rural y sin la suficiente consulta, resultando así currículos universalizantes, académicos, monótonos, excesivamente extensos y con muy poco margen de adaptación a las situaciones locales. La misma metodología. basada en materias aisladas, tiende a fomentar la fragmentación del aprendizaje. A lo anterior se une, de manera impactante y decisiva, la baja calidad humana y profesional predominante en el personal docente que tiene a su cargo la docencia en ese medio.

Los presupuestos para la educación probablemente han estado bajo una presión creciente en la década de los ochenta y continuará bajo la misma en la de los noventa. Esto es cierto tanto para los países industrializados como para los del tercer mundo en desarrollo.

En este contexto no se pueden fijar a priori modelos de planeamiento y administración. Por cada una de las diferentes situaciones que se encuentran en las zonas rurales de una región, habrá siempre un conjunto de opciones con distintos pesos y objetivos de crecimientos e, inevitablemente, con diversas posibilidades de desarrollo sociocultural.

Por otra parte, se debe poner énfasis en la necesidad de promover procesos educativos innovadores que se fundamenten en las propias necesidades y potencialidades de las diferentes poblaciones rurales de América Latina, a las que se debe servir en su propio contexto sociocultural y en los objetivos económicos y sociales a ser alcanzados como parte del desarrollo nacional en cada país. 


\section{Propuesta para mejorar la educación rural en América Latina}

Los programas de la educación rural deberían ser ofrecidos de acuerdo con nuevas políticas de acción en favor de ese sector poblacional. Los actuales sistemas educativos en el sector rural representan y reflejan la dependencia economica, social, política y cultural de la educación. En lugar de esto, deberian estar basados en el concepto integral del ser humano. en el desarrollo y en el papel de la educación en los cambios sociales. Entre los objetivos generales de nuevas políticas educacionales podemos señalar:

1. Crear condiciones favorables para conseguir ese desarrollo integral.

2. Estimular a los habitantes de los espacios rurales para que adquieran actitudes críticas, creativas, y de hermandad no ruralizantes.

3. Fortalecer la participación activa del sector rural en el diagnóstico de sus condiciones y para que tomen parte en la planificación. realización y evaluación de los procesos educativos en sus diferentes áreas.

Con base en todo lo anterior podriamos inferir que estamos en el momento propicio para que se promueva la formulación de políticas realistas específicas para el medio rural, como también para afirmar un proceso de planificación que asegure el desarrollo integrado de dicho medio de acuerdo a sus características.

Una reforma educativa debe ser propuesta de forma que una curricula nueva pueda ser desarrollada con fines de promover una mayor autonomia, igualdad de oportunidades y participación más amplia en los procesos educativos de la juventud rural. Las organizaciones campesinas existentes y los grupos de j6venes rurales pueden jugar un papael importante para auxiliar los cambios en la estructura social y en las instituciones educativas. La invasión de la cultura urbana también puede crear posibilidades para incorporar la juventud rural dentro de las corrientes de cambio.

Finalmente, toda la educación para el medio rural latinoamericano, además de estar integrada a los planes de desarrollo nacional, regional y local, deberá asegurar una atención al niño, al joven y al adulto en los aspectos más deficitarios y a garantizar la participación y beneficio de toda la población en el proceso de desarrollo. Merece una especial mención la educación de la mujer campesina en todos los programas que se lleven a cabo para el mejoramiento socioeconómico de tan importante sector humano. Apenas estamos comenzando a prestar la debida atención a la mujer, ya que habíamos relegado a un segundo plano su incorporación plena a la sociedad.

En lo que se refiere a las nuevas orientaciones sugeridas sobre la estructura, contenidos y métodos, deberían tenerse como principales los siguientes: 
- Concebir la nueva cstructura cducacional integrada por modalidades de educación, principalmente, de tipo extraescolar adaptadas a las condiciones de vida de la población y a la atención de sus necesidades básicas, siempre dentro de un concepto de educación permanente. El resultado de tal concepción de la estructura podría dar lugar a niveles y tipos de educación muy diferentes de los tradicionalmente considerados.

- Asociar la educación y la problemática del medio rural y, consecuentemente, adecuar los contenidos y, en general, todas las actividades educativas a dicha problemática y a los propósitos del desarrollo integrado del medio rural. Siendo el trabajo en el sector agropecuario uno de los clementos básicos de dicho desarrollo, la educación deberá realizarse en estrecha vinculación con aquel, sin menospreciar ni excluir los otros sectores del desarrollo rural.

- Impulsar una nucva formación y capacitación del educador (docentes y administradores) para que csté en condiciones de comprender el desarrollo integrado del medio rural y de participar activamente en el mismo, como animador y motivador de iniciativas comunitarias.

- Asegurar una participación consciente y una contribución efectiva de la comunidad, las que deberán ser consideradas en todo momento como recursos disponibles para la educación, y,

- Dentro de la orientación integradora, el educador no podrá tener el perfil tradicional que le ha habilitado solamente para actividades docentes en el aula. Será necesario definir distintos perfiles a partir de funciones de animación, promoción y docencia propiamente dichas.

\section{Conclusiones}

La función de la educación en el medio rural cs fundamental. No se puede concebir un programa eficaz de desarrollo rural sustentable si no se forma y capacita al habitante del campo para que cumpla su papel de protagonista y beneficiario de ese desarrollo, incluyendo a la mujer y a los jóvenes campesinos como piezas importantes de tan trascendental papel. No se trata solamente de darles al hombre y a la mujer conocimientos sino de capacitarles para trabajar y vivir mejor, así como, para desarrollar las habilidades que les permita cumplir mejor su papel de ciudadanos (a), como también saber adoptar una actitud positiva frente al desarrollo. Tampoco se trata solamente de facilitarles una preparación técnica sino, además, una formación cultural que complemente sus conocimientos y desarrolle sus capacidadesintelectuales; que les permita dominar la lectura y escritura, desenvolver sus conciencias críticas para situarse en el contexto de 
su medio social y entender su papel como participante de los procesos socioeconómicos.

Igualmente hay que conșiderar la formación de tipo sociopolítico, que les permita participar consciente y positivamente en la vida política y social de la comunidad local y de su país. Esto es lo que constituye una formación integral mediante la cual es posible crear un hombre y una mujer aptos para participar constructivamente en el desarrollo y luchar por su liberación del atraso, la ignorancia y la pobreza.

Desde luego, todo lo anterior implica una concepción integrada de la formación, de modo que unos aspectos actúen sobre los otros y se refuercen muluamente. Además, tiene que llevarse a cabo en función directa con las necesidades, intereses y problemas del habitante rural. Por último, esta formación no puede ser uniforme, ya que en la realidad del medio rural, existe una gran variedad de situaciones. De ahi que la educación del hombre y la mujer rurales deba situarse en el contexto del desarrollo nacional y en una situación donde opere una transformación en los demás aspectos de su vida, siendo ello indudablemente el elemento primordial de los cambios que deben operarse en las comunidades.

\section{Referencias}

Araujo, J.E.; Franco, A. (1985). Algunas políticas agrarias que puede retardar el crecimiento poblacional urbano. Desarrollo Rural en las Américas. San José, Costa Rica.

Berroteran, I. et al. (2000). Innovaciones en la educación técnica agropecuaria. Managua Nicaragua, INATEC-STOAS.

Conferencia Mundial sobre la Enseñanza de las Ciencias Agrícolas (1970. Copenhague, Dinamarca). UNESCO/FAO.

Contreras, V.; Roque, P. (1998). Sembrando esperanzas. La superación de la pobreza mediante el desarrollo de la inteligencia en la infancia y juventud. San José, Costa Rica, UNICEF-PNUD-iMAS.

Finchman, G.; Hernández, I. (1990). Movimiento indigenista, especificidad étnica y educación en América Latina. Nueva Educación (Arg.) 12(2): 40-46.

Labelle, T.J. (1984). Liberación, desarrollo y educación rural no formal. Anthropological and Education Quaterly 15(1): 80-93. 
Lewin, K.; Little, A.; Colclough, C. (1983). Efectos de la educación en los objetivos del desarrollo. Prospects (England) 13(3): 299-311.

Marull, J.D. (1978). Desarrollo rural en América Latina. Desarrollo Rural en las Américas 8(2): 125-136.

Reunión internacional sobre planificación educativa en paises multilingües. (1978). Conclusiones y recomendaciones. In Lenguaje y Ciencia (Perú)18:17-22.

Viñas- Roman, J.A. (1986). Generalidades sobre política de educación rural en la República Dominicana. Santo Domingo, Rep. Dom., Fundación para el Desarrollo de la Juventud Rural.

(1991). La juvenud en actividades de desarrollo rural. In Reunión del Consejo Asesor Iberoamericano de Juventudes Rurales. Santo Domingo, Rep. Dom. 